\title{
Staff perceptions of pod nursing on an acute mental health unit
}

\author{
Barb Pizzingrilli, Derek Christensen \\ Mental Health and Addictions Program, Niagara Health System, St. Catharines, Canada \\ Correspondence: Barb Pizzingrilli. Address: Mental Health and Addictions Program, Niagara Health System, St. \\ Catharines, Canada ON., L2S 0A9, Canada. Email: barb.pizzingrilli@niagarahealth.on.ca
}

Received: August 4, 2014

DOI : $10.5430 /$ jnep.v4n12p88

Accepted: September 24, 2014

URL: http://dx.doi.org/10.5430/jnep.v4n12p88

Online Published: October 9, 2014

\section{Abstract}

Objective: The Niagara Health System opened a new healthcare facility in 2013 with consolidation of inpatient mental health services at the new hospital site. The model of nursing care delivery was changed from primary nursing to pod nursing on one of the acute mental health units to better align care delivery with the physical lay-out of the unit that created challenges around long-standing care processes such as observational rounds, transfer of accountability, and team communication. Inefficient processes can decrease nursing visibility and accessibility and this can affect patient engagement and unit safety. The study sought to elicit staff perceptions of the impact of the model change for communication and gained efficiencies in completion of observational rounds and transfer of accountability. There is a gap in the literature related to pod nursing and this paper will inform decisions related to use of this model in acute mental health inpatient settings.

Methods: A seven-item questionnaire constructed by the authors was sent out by e-mail to the unit's 22 regular nursing staff nine months after implementation of the pod nursing model. The questionnaire was designed to elicit staff perceptions of the impact of the model change. The analytic sample consisted of results from 13 nursing staff who completed the questionnaire. Descriptive statistics were used to describe perception responses.

Results: Results of the survey identified overall staff agreement with pod nursing as an efficient model of care for the unit (92\%). The majority (84.6\%) of staff agreed that observational rounds were more manageable, the transfer of accountability process works well (100\%), and there is improved communication with their colleagues (61.5\%).

Conclusions: Pod nursing can be effectively implemented in the acute mental health setting and based on streamlined processes it can free up valuable nursing time to be visible and accessible on the unit, which can contribute to a safe and therapeutic milieu.

\section{Key words}

Mental health, Nursing models, Primary nursing, Pod nursing

\section{I ntroduction}

As healthcare efforts increasingly focus on the provision of safe, quality care, pressure exists for all staff to work efficiently and effectively in order to meet these goals. However, given the 24/7 presence of nursing staff, the pressure for 
this professional group can be even more intense. The topic of nursing care delivery models has been a significant area of interest within the literature for several decades. This is particularly true as a growing wealth of research evidence has emerged that identifies that the way in which nurses are organized to perform their care-related activities has a direct impact on patient outcomes ${ }^{[1]}$.

While safe, quality care is a consistent theme across all practice areas, these concepts are interpreted somewhat differently within mental health, which has implications for the choice of care delivery model within this specialty area. At the core of mental health care is the therapeutic relationship, from which all planning related activities arise. The nurse uses foundational interpersonal skills including empathy, trust, and respect to develop rapport with the patient and engage therapeutically in order to support the individual in moving forward to actualize self-identified goals. The therapeutic relationship is essential in supporting a safe milieu ${ }^{[2]}$ where access to care providers is required to address suicidal, homicidal, and other violence risk. The safety priority is to identify and act early to prevent near-miss suicide attempts and de-escalate violent behaviour to avoid harm ${ }^{[3]}$.

Traditionally, primary nursing was used as the care delivery model on the mental health inpatient units at the Niagara Health System (NHS), which involves accountability and responsibility for all care-related activities being assigned to a single nurse ${ }^{[4]}$. While primary nursing in its truest form identifies the Registered Nurse as the care provider, the NHS adapted the model to include both Registered Nurses and Registered Practical Nurses who are responsible for all aspects of patient care based on scope of practice. The use of a primary nursing model for care delivery aligns well with the goal of therapeutic engagement within the inpatient mental health setting.

The NHS opened a new healthcare facility in March 2013 with consolidation of inpatient mental health services from three sites to one with creation of two acute care units consisting of 20 beds (Unit B) and 24 beds (Unit M) respectively. In October 2013, approximately six months after moving into the new NHS healthcare facility, it became evident through conversations with staff on Unit $1 \mathrm{M}$ that the primary nursing model and related processes that had been in place prior to moving into the new hospital were not working well within the new physical environment of the unit. Staff cited the larger unit lay-out as being problematic related to patient care. Patient assignments completed by the Charge Nurses based on acuity, skill set, and scope of practice led to staff walking more to care for patients who often were located in separate areas of the unit. In addition, the larger unit resulted in more time required to complete the observational rounds that are ordered, on average, every 30 minutes for all 24 patients.

Staff identified that the transfer of accountability (TOA) and communication processes between shifts were also not working efficiently. The TOA consisted of all staff sitting together and listening to report on all 24 patients, which often took more than 30 minutes to complete. Staff then felt rushed to get their day organized including getting morning care activities done prior to patients having breakfast and beginning to use off-unit privileges. The larger unit design, the TOA process, general patterns of team communication, and observational rounds all contributed to lost nursing time. Process and design related factors that contribute to the inaccessibility of nursing staff to patients and their families may have an impact on therapeutic engagement and the proactive actions of staff in observing changes in patient presentation so that they can react to maintain safety ${ }^{[5]}$.

As a result, the team led by the Nurse Manager and Clinical Educator made the decision to look at other options for care delivery. A literature review was done to look at different models of care delivery, and in the preliminary stages of the search, pod nursing references were found. It was noted that there is a significant gap in the overall literature on this topic, with only a couple of articles found that were focused in medical/surgical areas of practice. There were two key articles on pod nursing that were reviewed: Donahue ${ }^{[6]}$ identified improved patient satisfaction and nurse vitality after implementation of pod nursing on a 38 bed cardiothoracic unit. By breaking the unit down into smaller pod areas, the nursing staff were able to reduce unnecessary walking around the larger unit, which increased their ability to monitor patients more closely and enhanced their visibility on the unit. Based on Donahue's work, pod nursing has the potential to 
increase therapeutic presence on the unit so that staff have the ability to "keep an eye on patients to reduce self-harm, violence, and manipulative and suicidal behaviour" ${ }^{[7]}$.

Friese et al. ${ }^{[8]}$ implemented pod nursing on a 32 bed medical/surgical unit. Similar to Donahue's ${ }^{[6]}$ findings, the authors found that nursing staff were able to collaborate more effectively and efficiently when working as partners in the pods and as a result satisfaction increased. These results highlight the benefits of pod nursing including enhanced collaboration, increased visibility of staff on the unit, and efficiency related to smaller geographic areas for staff to work in, which conserves valuable nursing time. The results from Friese and the other authors again highlight the efficiency that can be obtained from breaking the unit down into smaller areas of responsibility. This can increase nursing presence on the unit to improve safety.

While the number of articles on pod nursing is limited, the results obtained from the two that were reviewed aligned with the outcomes desired on Unit $1 \mathrm{M}$ so the decision was made to introduce pod nursing to the staff.

\section{I mplementation}

The Nurse Manager and Clinical Educator met with staff to explain pod nursing, and everyone expressed agreement in reorganizing the unit into three pods: Pods A, B, and C (see Figure 1).

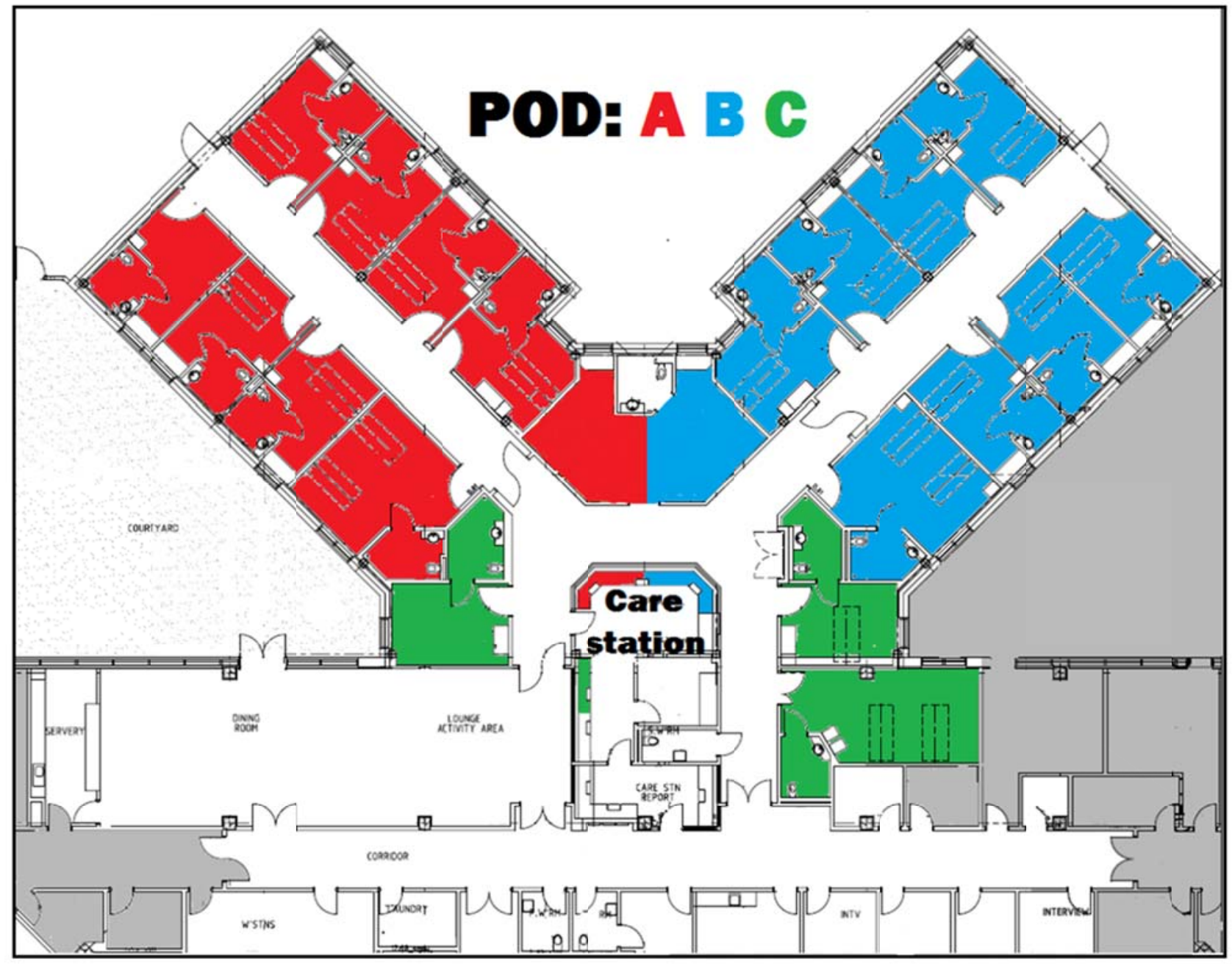

Figure 1. Unit M Pod Design 
Two of the pods consist of ten patients with a Registered Nurse (RN) and a Registered Practical Nurse (RPN) assigned and one pod of four patients assigned to a Registered Nurse. The four-bed pod consists of the higher acuity patients on the unit who often require more frequent observation and care that is consistent with a RN scope of practice. On day shift, each ten-patient pod aligns with a unit bedroom corridor, which enables a RN and RPN pair to be assigned to a corridor for patient related activities. The assignment of patients within the pod is negotiated between the RN and RPN, and wherever possible, continuity of care provider is encouraged. The RN and RPN staff schedules do not overlap exactly, so one of the staff may have been on previous shifts and will continue caring for specific patients rather than taking on new ones. The RN and RPN assume total care for the ten patients with collaboration around admissions, ongoing care, and discharges. Changes in acuity that previously would have resulted in the RPN transferring care to a RN can now be managed effectively within the pod. On afternoon and night shifts, the pods are realigned as staff numbers decrease, however, the integrity of the corridor assignment is maintained to the fullest extent possible during these times.

In order to facilitate documentation and communication, the team station was reorganized into the three pods (Pods A, B, C) with designated desk areas for staff. The ten-bed pods have two computers and one telephone and the four-bed pod has a computer and telephone for staff use. Staff were very enthusiastic about the reorganization of the team station and began supplying their respective pods with patient information and resource binders that could be easily accessed. The Clinical Educator developed coloured maps that showed the configuration of the pods across all shifts with Pod A being designated as red, Pod B as blue, and Pod C as green. Patient charts, Computerized Medication Administration Record (CMAR) binders, and the name of each pod-designated desk space were colour coordinated (see Figure 1).

The team station was designed and built with two slider windows that could be used for medication administration purposes. Prior to implementation of the pod model, the windows were rarely used and did not make sense given their location. However, with the pod implementation, one window now aligns with Pod A and the other with Pod B. Patients are encouraged to come to the windows at medication times and as needed. This has been more efficient for staff time as often numerous trips back to the medication room would be required for 'as needed' medication that was requested at the bedside. The Pod C staff use the two slider windows depending on where patients present for medications. Given the proximity of the Pod $\mathrm{C}$ bedrooms to the team station, the RN often administers medications at the bedside rather than have patients come to the windows.

The specific pod-related changes that have occurred on the unit are:

\section{Observational rounds}

The observational rounds are specific to each pod and occur for a maximum of ten patients, compared to completing rounds for all unit patients. This is more efficient than covering the whole unit for rounds, which often encompassed the entire 30 minute observation period. The location of the designated desk areas for Pods A and B facilitate clear sight lines of each patient corridor thus increasing observation of patients who are moving about the unit outside of regular observation periods. As staff are organized into pods that are smaller geographic areas, and with two nursing staff in Pods A and B, the staff have a better "situational awareness" and knowledge of where inpatients in their pods are at any given time.

\section{Transfer of accountability and communication}

The TOA process has been reorganized and now occurs within each pod with face-to-face communication between staff. At the end of the TOA, all unit staff come together with the Charge Nurse to complete a safety-focused huddle that ensures communication of patient risk-related issues such as suicidality, elopement, falls, or medical issues between pods. The huddles are repeated several times throughout each shift to ensure that all staff are aware of safety 'hot spots' on the unit.

\section{Promotion of teamwork and collaboration}

With the pod system there has been an increase in collaboration and teamwork as now the two large Pods, A and B, have a RN and RPN working closely in providing patient care versus the previous system with five nursing staff essentially 
working independent of each other. The support given to each other has been a positive aspect of the pod system especially when one of the staff is new to the unit or a novice nurse.

\section{Methods}

\subsection{Research design}

A descriptive survey design was chosen for this study using a questionnaire developed by the authors to elicit perceptions of nursing staff regarding the pod nursing change on Unit 1M, a 24-bed acute mental health unit at the Niagara Health System. Approval was obtained from the Niagara Health System Research Ethics Board.

Given the number of competing activities occurring within the Mental Health and Addictions Program related to new hospital transition, patient related outcomes were not identified as part of this work as it would not be possible to link these specifically to the pod nursing model.

\subsection{Sample}

The sample ( $\mathrm{n}=22)$ consists of 12 Registered Nurses (RNs) and 10 Registered Practical Nurses (RPNs) who regularly work on the unit.

\subsection{Questionnaire}

A seven-item questionnaire was constructed by the authors that uses a five-point Likert scale for staff to indicate agreement with each question ranging from strongly disagree (1) to strongly agree (5).

1) Pod nursing is a more efficient model of care for our unit.

2) Pod nursing has improved my communication with colleague(s).

3) Pod nursing makes observational rounds more manageable.

4) Patient acuity across assignments is an issue with pod nursing.

5) Completing transfer of accountability within the pods works well on the unit.

6) Pod nursing should remain as the nursing model on the unit.

7) Pod nursing has improved patient care on the unit.

\subsection{Data collection procedures}

An e-mail was sent out to the 22 staff in July 2014, nine months after implementation of the pod nursing model on Unit 1M. The e-mail asked each staff member to complete the questionnaire and a link took them directly to the online document through Survey Monkey ${ }^{[9]}$. Staff had one month to complete the questionnaire. A summary of staff responses was received by the authors at the end of the one month period from the online survey service.

Descriptive statistics were used to describe staff perceptions and were completed by the authors. Data is presented as observed rates and frequencies (see Table 1).

\section{Results}

Of the 22 nurses who received the questionnaire, 13 (59\%) responded (see Table 1). Ninety-two percent of respondents reported that pod nursing is a more efficient model of nursing care for Unit M and all respondents $(100 \%$; $n=13)$ agreed (n 
= 6) or strongly agreed $(n=7)$ that pod nursing should remain as the nursing model on the unit. The majority $(61.5 \% ; n=$ 8) agreed ( $(=5)$ or strongly agreed $(n=3)$ that communication between colleague(s) has improved with the introduction of pod nursing and all respondents $(100 \% ; n=13)$ agreed $(n=7)$ or strongly agreed $(n=6)$ that completing transfer of accountability within the pods works well. Furthermore, respondents $(84.6 \% ; n=11)$ also agreed $(n=7)$ or strongly agreed $(n=4)$ that pod nursing makes observational rounds more manageable.

Table 1. Staff Perceptions of Pod Nursing

\begin{tabular}{|c|c|c|c|c|}
\hline \multirow{2}{*}{ Questions } & \multicolumn{2}{|c|}{ Strongly Disagree, Disagree or Neutral } & \multicolumn{2}{|c|}{ Strongly Agree or Agree } \\
\hline & $n$ & $\%$ & $n$ & $\%$ \\
\hline 1. More efficient model of care. & 1 & 7.7 & 12 & 92.3 \\
\hline 2. Improved communication with colleague(s). & 5 & 38.5 & 8 & 61.5 \\
\hline 3. Observational rounds more manageable. & 2 & 15.4 & 11 & 84.6 \\
\hline 4. Acuity of patients is an issue. & 9 & 69.2 & 4 & 30.8 \\
\hline 5. Transfer of accountability works well. & 0 & 0 & 13 & 100 \\
\hline 6. Should remain as the nursing model. & 0 & 0 & 13 & 100 \\
\hline 7. Improved patient care on the unit. & 2 & 15.4 & 11 & 84.6 \\
\hline
\end{tabular}

\section{Discussion}

Moving into a new hospital provided the opportunity to examine long-standing processes that no longer aligned with the new unit designs, which are more spacious and require more walking to complete care related activities. The introduction of pod nursing on Unit 1M, which is an acute mental health unit, has resulted in improved efficiencies for the nursing staff. Communication is enhanced between the pod partners who work collaboratively to care for assigned patients rather than working alone. Observational rounds, and the transfer of accountability process have been identified as improved with the pod nursing model largely due to reduced time required to complete these activities, which enables staff to focus on therapeutic activities that will contribute to an environment of safety.

Staff feedback since the implementation of pod nursing in October 2013 has identified one area of concern that was included in the staff questionnaire. While all efforts are made to place the higher acuity patients in the four-bed pod adjacent to the team station, this is not always possible if the number of acute patients exceeds the four bed capacity. The acute patients are then placed in another pod. However, availability of more beds in one pod than the other may disproportionately increase the acuity. While approximately two-thirds of respondents $(69.2 \% ; n=9)$ disagreed $(n=5)$ or strongly disagreed $(n=4)$ that patient acuity across assignments is an issue with pod nursing, there were four respondents (30.8\%) who agreed with this statement. There may be times when the acuity varies considerably across the pods, but with better communication, presence of staff within the pods at all times, and ability to reassign patients between nursing partners, the care of these higher need patients can be effectively managed.

The cost of implementing pod nursing on the unit of interest was minimal as the unit had been designed in a manner that was conducive to the pod model. However, other hospitals interested in implementing pod nursing may require physical lay-out changes that could be cost prohibitive. Any design changes and the costs associated with these would need to be factored into any decisions related to implementation of pod nursing.

The response rate was reasonable for the questionnaires, but there could still be a bias if the staff members who did not respond represent different views with respect to pod nursing.

The introduction of pod nursing was done during the first year of transition to the new hospital. There were many competing priorities that could impact patient satisfaction and safety dimensions of care. Therefore, the decision was made 
to not capture these items as part of the project evaluation. For other hospitals interested in implementing pod nursing it would be important to ensure a robust evaluation strategy that includes both patient, family and staff components.

The staff results indicate that implementation of pod nursing on the acute mental health unit has been effective in improving communication and processes such as observational rounds and transfer of accountability. Pod nursing can be implemented in both mental health and non-mental health units though physical design and lay-out may require some modifications to the model suggested within this paper.

\section{Conclusion}

The results from this paper will contribute to the literature on pod nursing and on the use of this model in acute mental health inpatient settings. Further research needs to be conducted to explore the potential of pod nursing in other settings beyond the limited work that has been done to date.

\section{Acknowledgement}

The authors would like to thank all of the Unit $1 \mathrm{M}$ staff who enthusiastically embraced this significant model change. Without the dedication and commitment of all the staff, the changes could not have been actualized.

\section{References}

[1] Dubois, C.A., D’Amour, D., Tchouaket, E., Clarke, S., Rivard, M., Blais, R. Associations of patient safety outcomes with models of nursing care organization at unit level in hospitals. International Journal of Quality in Health Care. 2013; 25(2): 110-117. http://dx.doi.org/10.1093/intqhc/mzt019

[2] Muir-Cochrane, E., Oster, C., Grotto, J., Gerace, A., Jones, J. The inpatient psychiatric unit as both a safety and unsafe place: Implications for absconding. International Journal of Mental Health Nursing. 2013; 22: 304-312. http://dx.doi.org/10.1111/j.1447-0349.2012.00873

[3] Jeffs, L., Rose, D., Macrae, C., Maione, M., \& Macmillan, K.M. What near misses tell us about risk and safety in mental health care. Journal of Psychiatric and Mental Health Nursing. 2012; 19: 430-7. PMid:22070194 http://dx.doi.org/10.1111/j.1365-2850.2011.01812.x

[4] Tiedeman, M.E., \& Lookinland, S. Traditional models of care delivery: What have we learned? Journal of Nursing Administration. 2004; 34: 291-97. PMid:15190224 http://dx.doi.org/10.1097/00005110-200406000-00008

[5] Kanerva, A., Lammintakanen, J., \& Kivinen, T. Patient safety in psychiatric inpatient care: A literature review. Journal of Psychiatric and Mental Health Nursing. 2013; 20(6): 541-8. http://dx.doi.org/10.1111/j.1365-2850.2012.01949.x

[6] Donahue, L. Pod design for nursing assignments: Eliminating unnecessary steps and increasing patient satisfaction by reconfiguring care assignments. American Journal of Nursing. 2009; 109(11): 38-40. PMid:19826335 http://dx.doi.org/10.1097/01.NAJ.0000362019.47504.9d

[7] Salzmann-Erikson, M., \& Eriksson, H. Panoptic power and mental health nursing - space and surveillance in relation to staff, patients, and neutral places. Issues in Mental Health Nursing. 2012; 33: 500-504. http://dx.doi.org/10.3109/01612840.2012.682326

[8] Friese, C. R., Grunawalt, J. C., Bhullar, S., Bihlmeyer, K., Chang, R., Wood, W. Pod nursing on a medical/surgical unit: Implementation and outcomes evaluation. Journal of Nursing Administration. 2014; 44(4): 207-211. http://dx.doi.org/10.1097/NNA.0000000000000051

[9] Pizzingrilli, B., \& Christensen, D. Pod Nursing Survey. 2014. Available from: http://www.surveymonkey.com/s/3CVXW37 